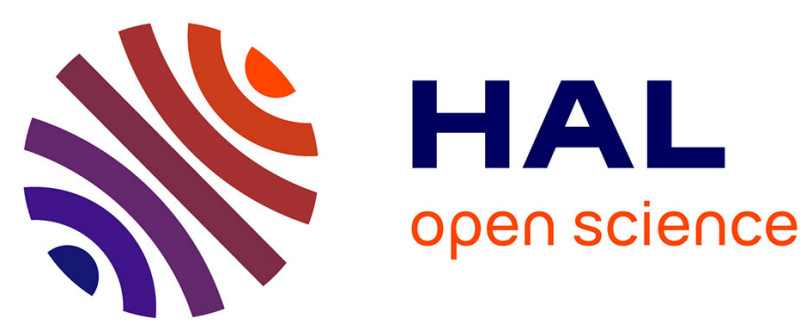

\title{
Fundamental thermal-hydraulic continuous pulp digester model with grade transition
}

\author{
Sharad Bhartiya, Pascal Dufour, Francis J. Doyle Iii
}

\section{To cite this version:}

Sharad Bhartiya, Pascal Dufour, Francis J. Doyle Iii. Fundamental thermal-hydraulic continuous pulp digester model with grade transition. American Institute of Chemical Engineers, 2003, 49 (2), pp.411-425. 10.1002/aic.690490212 . hal-00352339v2

\section{HAL Id: hal-00352339 \\ https://hal.science/hal-00352339v2}

Submitted on 21 Jan 2009

HAL is a multi-disciplinary open access archive for the deposit and dissemination of scientific research documents, whether they are published or not. The documents may come from teaching and research institutions in France or abroad, or from public or private research centers.
L'archive ouverte pluridisciplinaire HAL, est destinée au dépôt et à la diffusion de documents scientifiques de niveau recherche, publiés ou non, émanant des établissements d'enseignement et de recherche français ou étrangers, des laboratoires publics ou privés. 
This document must be cited according to its final version which is published in a journal as:

S. Bhartiya ${ }^{1}$, P. Dufour ${ }^{1}$, F.J. Doyle III ${ }^{1}$,

"Fundamental thermal-hydraulic

continuous pulp digester model with grade transition", American Institute of Chemical Engineers (AIChE) Journal ISSN: 0001-1541, 49(2), pp. 411-425, 2003. http://dx.doi.org/10.1002/aic.690490212

All open archive documents of Pascal Dufour are available at: http://hal.archives-ouvertes.fr/DUFOUR-PASCAL-C-3926-2008

The professional web page ( $\mathrm{Fr} / \mathrm{En}$ ) of Pascal Dufour is: http://www.lagep.univ-lyon1.fr/signatures/dufour.pascal

1

University of Delaware, Department of Chemical Engineering, Newark, DE 19716, USA

http://www.udel.edu 


\title{
FUNDAMENTAL THERMAL-HYDRAULIC CONTINUOUS PULP DIGESTER MODEL WITH GRADE TRANSITION
}

\author{
Sharad Bhartiya, Pascal Dufour and Francis J. Doyle III ${ }^{1}$ \\ Department of Chemical Engineering, University of Delaware, Newark DE 19716
}

This paper presents a detailed fundamental model of a continuous pulp digester developed for simulation and control. Most modeling efforts in the past have either emphasized reaction kinetics and energy transfer assuming a prescribed flow behavior, or modeled digester hydraulics with simplified reaction kinetics. In this work, we augment the well-known extended Purdue model (Wisnewski et al., 1997) by incorporating axial momentum transport, an immediate consequence of which is ability to simulate chip level and its impact on the Kappa number profile. An essential contribution is the ability to model chip compaction. Interaction between digester hydraulics and kinetics is illustrated via simulation examples. Examples include scenarios that show incipient plugging of the digester vessel. The current work also explores and illustrates the use of higher order finite-difference methods to reduce numerical dissipation and dispersion. Ability to describe chip velocities enables rigorous tracking of transition front within the digester during feedstock grade transitions. Operational difficulties encountered during hardwood to softwood transition are explained using the model.

\section{Topical Heading}

Process Systems Engineering

\section{Keywords}

Digesters (Paper making) - mathematical models; dynamic models; momentum balances; grade transitions; temperature distribution

\section{Introduction}

Pulping mills convert wood chips to pulp suitable for paper production by displacing lignin from cellulose fibers. The conversion is achieved through a combination of strategies involving thermal, chemical and mechanical degradation of the wood chips. Continuous Kraft processes use large, vertical, tubular reactors called digesters

\footnotetext{
${ }^{1}$ Corresponding author: Fax (302) 831 1048; Phone (302) 831 0760; email fdoyle@udel.edu
} 
where the chips react with an aqueous solution of sodium hydroxide and sodium sulfide, known as white liquor, at elevated temperature. Most continuous digesters consist of three basic zones: an impregnation zone, a cooking zone and a wash zone. A schematic of a single vessel digester is shown in Figure 1. White liquor and pre-steamed chips are introduced at the top of the digester into the impregnation zone where the liquor penetrates the wet chips. However, the majority of the delignification reaction occurs only after the two streams flow downward into the subsequent cooking zone where the mixture is heated to reaction temperatures achieved by liquor circulation through external heaters. The spent liquor is withdrawn from the digester at extraction screens located at the end of the cook zone while the cooked chips continue the downward journey to the wash zone. Here, the chips are washed by the countercurrent flow of cold, dilute liquor. This effectively quenches the delignification reaction. The quality of the resulting pulp is described by Kappa number (Kappa \#), which is a measure of the residual lignin content. A typical control objective of digester operation is to minimize variation in the Kappa \# from a prescribed value. Continuous digesters present challenging problems in modeling and control. From a modeling perspective, the inter-play between heat, mass and momentum transport during the thermal-hydraulic degradation of the wood chips creates rich dynamic behavior. For example, softening of the chips, as cooking proceeds, causes them to compact more densely, which in turn affects the chip velocity profiles.

The capital-intensive nature of the pulp \& paper industry mandates development of operating strategies that use enhanced control, soft-sensing and fault diagnosis methodologies, which are usually predicated on availability of a process model. A significant effort has been directed to development of first-principles models that describe the thermal-hydraulic degradation of wood chips in the continuous pulp digester. Most fundamental continuous digester models in literature can be classified into two broad categories depending on the attributes they emphasize, 1) pulping chemistry; and 2) hydraulic description of the chip and liquor streams. Smith and Williams (1974) developed kinetic models to describe delignification of chips based on the following form:

$$
R_{s, i}=-\left(k_{1, i}(T) C_{O H}^{a}+k_{2, i}(T) C_{O H}^{b} C_{H S}^{c}\right)\left(C_{s, i}-C_{s, i}^{\infty}\right)
$$

where the temperature dependence of the rate constants are calculated using the Arrhenius law:

$$
k_{i, j}(T)=A_{i, j} \exp \left(\frac{-E_{i, j}}{R T}\right)
$$


The wood substance was classified as high and low reactive lignin, cellulose, galactoglucomannan and araboxylan. Indices $i$ and $j$ refer to the two rate constants and the five solid components in the wood chips, respectively. Model constants $\left(a, b, c, A_{i, j}, E_{i, j}\right)$ were evaluated by trial and error using pulping chemistry data. Smith and Williams then approximated the digester by a series of continuous stirred tank reactors (CSTRs) to obtain dynamic mass balances for the main components participating in pulping reactions. Each CSTR was assumed to contain three phases: 1) a solid phase consisting of the wood substance; 2) an entrapped liquor phase comprising of liquor in the pores of the wood chips; and 3) a bulk free liquor phase surrounding the wood chips. The resulting digester model is referred to as the Purdue model. The major contribution of the Purdue model was development of a framework upon which subsequent models could be based. Christensen et al. (1982) modified the Purdue model by incorporating improved kinetic parameters (based on optimization search) valid over a larger range of wood species. The improved model predicted free liquor concentration profiles and blow-line Kappa \# for an industrial digester. Gustafson et al. (1983) developed alternate kinetic expressions to describe the delignification reaction. They use fewer number of wood and liquor components, and make use of functional relationships between rate of consumption of carbohydrates and lignin. Greater emphasis is paid to penetration of liquor inside the chips.

Kayihan et al. (1996) presented a Weyerhaeuser benchmark model for a two-vessel Kamyr digester with separate impregnation and reaction vessels. The model assumed two phases: nonporous solid and free liquor, which exist in thermal equilibrium. The kinetic model of Christensen et al. (1982) was adopted to describe dynamic mass balances. The resulting set of partial differential equations (PDEs) is discretized spatially and the model solved as a system of ordinary differential equations (ODEs). Extensions of the Purdue model have been presented by Wisnewski et al. (1997). They redefined mass concentrations and volume fractions that allowed relaxation of certain assumptions in the previous Purdue model leading to higher model fidelity. The extended Purdue model compared favorably and captured the range of behaviors displayed by the original Purdue model as well as the Weyerhaeuser benchmark 2-vessel digester model.

In all of the preceding works, a steady, unchanging compaction profile is assumed. Further, these models fail to provide a dynamic description of chip level, an important control variable. This is a necessary consequence of absence of momentum transport modeling. On the other hand, hydraulic description based models assume simpler 
chemistry, constraining the validity to narrower ranges of Kappa \#. Härkönen (1987) presented a detailed description of chip and liquor flow dynamics, and compaction, in addition to mass and energy transport. Constitutive equations that described flow resistance were developed experimentally. The resulting model was a system of two-dimensional PDEs that were solved at steady state. Simplifying assumptions were made with regards to the reaction kinetics and delignification in wood chips is ignored. Nevertheless, Härkönen's work laid the foundation for integrating digester hydraulics with existing models based on mass and energy transport. Michelsen (1995) proposed an integration of the works by Christensen et al. (1982) and Härkönen (1987) to develop a detailed digester model from mass, energy and axial momentum transport balances. The model assumes solid, entrapped liquor and free liquor phases in the digester and is described by a complex set of algebraic equations and PDEs. It is solved numerically using a staggered grid finite difference approach. While chip and liquor flow dynamics are described in detail, simplifications are made in reaction kinetics, which constrain the validity of the model for Kappa \# within the range of 50 and 150. A key result of Michelsen's model was ability to study the effect of flow behavior and compaction on Kappa \#. Further, the study suggested residence time control could yield improved Kappa \# control.

The model developed in this article is a direct integration of the works of Wisnewski et al. (1997) and Michelsen (1995) that overcomes some of the deficiencies described above. Thus, the detailed kinetics and mass/energy transport of the industrially established extended Purdue model are augmented with Michelsen's momentum transport description, yielding a thermal-hydraulic model of the continuous digester. Incorporation of momentum allows rigorous simulation of phenomena distinguished by interactions between the flow mechanics of the chips and free liquor and the delignification kinetics. These include digester level, production rate change, feedstock grade transition, as well as incipient plugging of digester. The next section presents the model and develops the model equations including a brief discussion on numerical issues. Next, the model features are illustrated through simulations and parametric sensitivity followed by an extension of the model to simulate grade transitions. Finally, the work is summarized and further utility of the model is discussed. 


\section{Model Derivation}

The proposed model is based on a distributed parameter approach, where the axial distribution of transport properties occurs due to convection of the chips and liquor streams. Entry and exit of external streams, wall, and cross-flow effects (such as, at extraction screen and heater inlets and outlets) are likely to cause radial gradients in temperature and concentration profiles (He et al., 1998). However, all radial gradients are neglected as a simplifying assumption. The temporal variations within each infinitesimal control volume are described by conservation statements, resulting in a set of coupled nonlinear PDEs. External flows from heaters and extraction screens are modeled as flows entering and/or leaving the control volume. The remainder of the section outlines the transport equations and constitutive rules used. The nomenclature and definitions used in the present document closely follows that of Wisnewski et al. (1997).

\section{Physical Description}

As in the Purdue model, each control volume is assumed to contain three phases: solid phase, entrapped liquor phase, and free liquor phase. The entrapped liquor phase resides within the pores of the wood chips where it reacts with the solid substance. However, dynamic and thermal equilibria are assumed between these two phases. In this work, the combined solid and entrapped liquor phases will be referred to as the chip phase. A schematic of an infinitesimal slice of the digester with volume $\Delta V$ is show in Figure 2. The results presented later assume control volumes (CVs) with fixed cross-sectional areas, $A$. Inventory of each phase at a fixed vertical location in the digester is measured using the following volume fraction definitions,

$$
\eta=\frac{\text { volume of free liquor phase in } \Delta V}{\text { volume of } \Delta V}\left[\frac{\Delta V_{f}(z)}{\Delta V(z)}\right]
$$

The remainder of the control volume is assumed to be occupied by the chip phase. Thus,

$$
(1-\eta)=\frac{\text { volume of chip phase in } \Delta V}{\text { volume of } \Delta V}\left[\frac{\Delta V_{c}(z)}{\Delta V(z)}\right]
$$

Compaction profile along the length of the digester is influenced by the volumetric flowrates of the chip and free liquor phases as well as the degree of cooking. The extended Purdue model assumed the compaction profile to be a fixed parameter of the model. The current work explicitly models chip compaction. Since chip phase volume 
consists of the solid wood substance and entrapped liquor, one can define the fraction of the chip phase occupied by entrapped liquor as the chip porosity,

$$
\mathcal{E}=\frac{\text { volume of entrapped liquor phase in } \Delta V}{\text { volume of chip phase in } \Delta V}\left[\frac{\Delta V_{e}(z)}{\Delta V_{c}(z)}\right]
$$

Equivalently,

$$
(1-\varepsilon)=\frac{\text { volume of solid phase in } \Delta V}{\text { volume of chip phase in } \Delta V}\left[\frac{\Delta V_{s}(z)}{\Delta V_{c}(z)}\right]
$$

As shown in Figure 2, the chip phase enters the digester slice with volumetric flowrate $\dot{V}_{c}(z)$, and exits at a rate of $\dot{V}_{c}(z+\Delta z)$. Free liquor flows cocurrently, with the chip phase, in the cooking zone and countercurrently in the wash zone. Volumetric flow of free liquor is defined by $\dot{V}_{f}$. Chip and free liquor phase velocities, $v_{c}$ and $v_{f}$, are defined using, respectively:

$$
\begin{aligned}
& \dot{V}_{c}=A(1-\eta) v_{c} \\
& \dot{V}_{f}=A \eta v_{f}
\end{aligned}
$$

Specie concentrations employed in the conservation equations make use of definitions formulated by Wisnewski et $a l$. as opposed to the earlier versions of Purdue model. The solid component concentration is defined on a chip volume basis as follows,

$$
\rho_{s, i}=\left[\frac{\text { mass of solid component } i \text { in chip }}{\text { chip volume }}\right]
$$

similarly, for entrapped and free liquor concentrations, respectively

$$
\begin{gathered}
\rho_{e, i}=\left[\frac{\text { mass of entrapped liquor component } \mathrm{i} \text { in chip }}{\text { entrapped liquor volume in chip }}\right] \\
\rho_{f, i}=\left[\frac{\text { mass of freed liquor component } \mathrm{i}}{\text { free liquor volume }}\right]
\end{gathered}
$$

\section{Solid Phase Mass Continuity}

The solid phase is assumed to consist of five components: high-reactive lignin $\left(s_{1}\right)$; low-reactive lignin $\left(s_{2}\right)$; cellulose $\left(s_{3}\right)$; araboxylan $\left(s_{4}\right)$; and galactoglucomannan $\left(s_{5}\right)$. Physical transport as well as the rate at which they react with the 
liquor determines the reactant concentrations. Performing a component balance for the solid phase at location $z$ yields the following,

$$
\frac{\partial \rho_{s, i}}{\partial t}=-v_{c} \frac{\partial \rho_{s, i}}{\partial z}+\hat{R}_{s, i}
$$

where $\rho_{s, i}$ is defined in Equation (8). $\hat{R}_{s, i}$ represents the rate of consumption of mass of solid $i$ per chip volume. The reaction rate used in the current work is based on the kinetic model developed by Christensen et al. (1982),

$$
\hat{R}_{s, i}=-e_{f}\left(k_{1, i} \rho_{e, 1}+k_{2, i} \rho_{e, 1}^{1 / 2} \rho_{e, 3}^{1 / 2}\right)\left(\rho_{s, i}-\rho_{s, i}^{\infty}\right)
$$

where $e_{f}$ is the reaction rate effectiveness factor and used to adjust the kinetics for different wood species. However the main use of the effectiveness factor lies in tuning the model outputs to observed digester behavior. $\rho_{s, i}^{\infty}$ denotes the concentration of component $i$ entering the digester that is inert. Reaction rate constants $k_{1, i}$ and $k_{2, i}$ are determined using the Arrhenius law. Values of kinetic parameters for hardwood and softwood are summarized in Wisnewski et al. (1997).

\section{Entrapped Liquor Phase Mass Continuity}

It is assumed that the chips are saturated with water upon entering the digester. The water then mixes with reactants from free liquor that diffuse into the entrapped liquor and the dissolved reaction products from the solid phase. The entrapped liquor is, thus, an aqueous solution with six components: active effective alkali (EA) $\left(e_{1}\right)$; passive EA $\left(e_{2}\right)$; active hydrosulfide (HS) $\left(e_{3}\right)$; passive HS $\left(e_{4}\right)$; dissolved lignin $\left(e_{5}\right)$ and dissolved carbohydrates $\left(e_{6}\right)$. The entrapped liquor components are consumed during delignification of the solids and replenished in the cook zone by the free liquor via diffusion. A component balance yields,

$$
\frac{\partial \rho_{e, i}}{\partial t}=-\frac{\rho_{e, i}}{\varepsilon} \frac{\partial \varepsilon}{\partial t}-\frac{v_{c}}{\varepsilon} \frac{\partial\left(\varepsilon \rho_{e, i}\right)}{\partial z}+D\left(\rho_{f, i}-\rho_{e, i}\right)+\hat{R}_{e, i}
$$

$\rho_{e, i}$ represents concentration of entrapped liquor components defined as mass of component $i$ in an elementary control volume per entrapped liquor volume (see Equation (9)). Porosity of chips, $\varepsilon(z)$, is computed by comparing the density of solid phase components defined on chip volume basis with density of solid wood substance, $\bar{\rho}_{s}$, as follows, 


$$
\varepsilon(z, t)=1-\frac{\sum_{i=1}^{5} \rho_{s, i}(z, t)}{\bar{\rho}_{s}}
$$

The third term on the right hand side of Equation (13) models the diffusion of component $i$ from the free liquor phase to entrapped liquor phase. Early Purdue developers (Smith and Williams, 1974; Christensen et al., 1982) derived the following correlation for the mass diffusion rate (defined on a volume basis), $D$, using experimental data for Kraft cooking presented in McKibbins (1960),

$$
D=6.1321 \sqrt{T_{c}} \exp \left(\frac{-4870}{1.98 T_{c}}\right) \quad\left[\frac{1}{\min }\right]
$$

Simulation results presented later are based on this correlation. Reaction rates, $\hat{R}_{e, i}$ and $\hat{R}_{s, i}$, are related via stoichiometric coefficients, $b_{i, j}$,

$$
\hat{R}_{e, i}=\sum_{j=1}^{5} b_{i, j} \hat{R}_{s, j}
$$

The boundary conditions for the solid and entrapped phase continuity equations are fixed by the wood specie and the degree of impregnation.

In their work, Wisnewski et al. (1997) assume that the solids react and dissolve into the entrapped phase, contributing only mass and not volume. The newly created volume then draws in free liquor from the neighboring free liquor phase. However, as discussed previously, this assumption violates volume continuity needed for calculation of compaction, $\eta$, and free liquor volumetric flowrates, $\dot{V}_{f}$. Thus, only the diffusive mechanism phase is retained as the means of mass transfer from the free liquor to the entrapped phase. Since, the entrapped liquor phase is incompressible, neglecting the vacuum effect amounts to assuming that the reacted solid components retain their density as in unreacted form in the solid phase.

\section{Free Liquor Phase Mass Continuity}

The free liquor phase is an aqueous solution of the same six components as in the entrapped liquor phase. Apart from bulk transport along the digester, free liquor concentrations are determined by the rate of diffusion to the entrapped liquor and mixing with external liquor streams. A component balance around the infinitesimal control volume gives the following, 


$$
\frac{\partial \rho_{f, i}}{\partial t}=-v_{c} \frac{\partial \rho_{f, i}}{\partial z}-D \varepsilon \frac{(1-\eta)}{\eta}\left(\rho_{f, i}-\rho_{e, i}\right) \pm \rho_{f, i, e x t} \frac{\dot{V}_{e x t}}{\Delta V_{f}}
$$

Subscript ext refers to external streams (for example, heater circulation streams). The positive sign in the last term refers to external flows entering the control volume and negative for exiting flows. $\rho_{f, i}(z)$ represents concentration of component $i$ per volume of free liquor (see Equation (10)). The rate of diffusion of free liquor to entrapped phase is equal to that entering entrapped phase in Equation (13) but expressed on a free liquor volume basis. The boundary conditions for the free liquor continuity equations are provided by the concentrations of white liquor components in the cook zone and concentration of wash liquor components in the wash zone.

\section{Chip and Free Liquor Thermal Energy Balances}

Wisnewski et al. (1997) use mixing rules based on weighted averages to determine heat capacities of the entrapped and free liquor phases, namely, $C_{P e}$ and $C_{P f}$, respectively. The same approach is retained in the current work. Below, energy balance equations modified to accommodate variable compaction are presented for the chip and free liquor phases and describe the temporal variation of the chip phase temperature $T_{c}$ and free liquor temperature, $T_{f}$.

$$
\begin{aligned}
\left(C_{P_{s}} M_{s}+C_{P_{e}} M_{e} \varepsilon\right) \frac{\partial T_{c}}{\partial t}=- & T_{c}\left(C_{P_{s}} \frac{\partial M_{s}}{\partial t}+C_{P e} \frac{\partial\left(M_{e} \varepsilon\right)}{\partial t}\right)-v_{c} \frac{\partial}{\partial z}\left[\left(C_{P_{s}} M_{s}+C_{P_{e}} M_{e} \varepsilon\right) T_{c}\right]+\Delta H_{R} \sum_{i=1}^{5} \hat{R}_{s, i} \\
& +U\left(T_{f}-T_{c}\right)+D \varepsilon D_{E}
\end{aligned}
$$

Heat released by the exothermic reactions between the entrapped liquor and solid phase components is modeled using the heat of reaction $\Delta H_{R}$. The heat transfer coefficient $U$ models the convective heat transfer between the chips and free liquor phases. $D_{E}$ represents the amount of energy transferred due to diffusion of components between the entrapped liquor and free liquor phases. The direction of diffusion is dependent on the concentration gradient. Thus,

$$
\left.D_{E}=\sum_{i=1}^{4} \rho_{f, i}-\rho_{e, i}\right) C_{P_{l}} T_{i}+\sum_{i=5}^{6}\left(\rho_{f, i}-\rho_{e, i}\right) C_{P_{s}} T_{i}
$$

where,

$$
T_{i}= \begin{cases}T_{f} & \text { if } \rho_{f, i}>\rho_{e, i} \\ T_{c} & \text { if } \rho_{f, i}<\rho_{e, i}\end{cases}
$$

A similar balance for the free liquor phase yields, 
$C_{P f} M_{f} \frac{\partial T_{f}}{\partial t}=-T_{f} \frac{\partial}{\partial t}\left(C_{P f} M_{f}\right)-v_{f} \frac{\partial}{\partial z}\left(C_{P f} M_{f} T_{f}\right)-U \frac{(1-\eta)}{\eta}\left(T_{f}-T_{c}\right)-\frac{D \varepsilon(1-\eta) D_{E}}{\eta} \pm \frac{\dot{V}_{e x t} M_{e x t} T_{e x t}}{\delta V_{f}}$

The term $\frac{D \varepsilon(1-\eta) D_{E}}{\eta}$ represents the energy transfer with the chip phase per volume of diffusing mass. The last term represents the energy transfer due to bulk motion of incoming/exiting external streams. Temperatures of wood chip charge, entering white liquor and entering wash liquor temperatures fix the boundary conditions needed for Equations (18) and (20). Equations (1) to (20) are based on the work by Wisnewski et al. (1997) but modified to account for chip compaction and momentum balances. The reader is referred to that work as the source for all parameters and constants (for example, values of heat transfer coefficient, diffusion coefficient, etc.) used in the simulation results presented in the next section.

\section{Chip Phase Volume Continuity}

As discussed previously, progressive cooking of chips cause them to soften, thereby changing shape but not volume. The only mechanism that affects compaction of chips in a given control volume is the convective flows of chip phase and liquor. Thus, a volume balance for the elemental volume, $\Delta V$, yields the following compaction equation,

$$
\frac{\partial \eta}{\partial t}=\frac{\partial}{\partial z}\left[(1-\eta) v_{c}\right]
$$

The compaction equation is central in providing insights to incipient digester plugging discussed later. Michelsen's model includes axial dispersion in addition to convective flow of chips. However, subsequent steady-state analysis shows that the dispersion has a negligible effect on compaction. Michelsen also makes a degree of freedom argument to demonstrate how volume continuity follows from mass continuity. Boundary condition for Equation (21) are fixed by compaction of chips in a top section.

\section{Overall Digester Volume Continuity}

Equation (21) provides information on the fraction of the control volume occupied by chips. To satisfy overall digester volume continuity, the remainder of the volume must be occupied by free liquor. This in turn, prescribes the volumetric flowrate of free liquor as follows,

$$
\nabla_{z}\left(\dot{V}_{c}+\dot{V}_{f}\right)=0
$$


where the incoming and exiting external flows are included in the liquor flowrate. Equation (22) is a static constraint expressing incompressibility of the contents of a control volume in the digester. It states that the sum of all flowrates entering a control volume of fixed size equals the sum of all flowrates exiting the volume. In the extended Purdue model (Wisnewski et al., 1997), bulk flow of free liquor into the entrapped phase by the vacuum effect decreases the flowrate of the exiting free liquor stream. However, no corresponding volume increase occurs in the chip phase thereby violating the incompressibility assumption. We assume that the rate of extraction of the liquors from the cook and wash zone is perfectly controlled. These values are then used to compute the volumetric flow distribution using Equation (22).

\section{Pressure Distribution in the Free Liquor Phase}

The free liquor pressure distribution, $p_{f}(z)$, is mainly dictated by the hydrostatic head in the digester. Depending on the degree of cooking, the chip phase at a given location, $z$, has a certain Kappa \# associated with it. Härkönen (1987) performed experimental studies to evaluate the effect of cooking on the compactability of chips and then defined a chip pressure, $p_{c}$, which is the force that acts through the inter-chip contact points over a reference area. As noted by Michelsen (1995), the chips become softer during cooking enabling them to pack more tightly. The softening of chips is reflected by reduced chip pressure. Härkönen's work (1987) also suggests that while the chips become softer during cooking, they change shape but no appreciable change in volume occurs. Calculation of the free liquor pressure distribution in presence of liquor velocity transients is a non-trivial task. Iterative methods (ex. SIMPLE algorithm, Patankar, 1980) exist in literature, which simultaneously arrive at a distribution of pressure and velocity fields such that the momentum and continuity equations are satisfied. However, the approach used here is to neglect free liquor momentum dynamics and utilize the resulting momentum equation to prescribe the pressure distribution. Michelsen (1995) suggests that this approach may be reasonable, since the period of free liquor velocity transients will be short due to incompressibility implied by Equation (22).

Various forces affecting the system determine the free liquor pressure. Given the large capacity and height of the digester, gravity plays an important role in determining the liquor pressure. The remainder of the forces arise as a result of the viscous resistance faced by the chips as they flow through the liquor phase with a non-zero relative 
velocity. Härkönen, (1987) developed expressions for the resistive force based on the Ergun equation. He defined the resistive force as,

$$
F_{\Lambda}=\Lambda v_{c, f}
$$

where the coefficient of viscous friction is,

$$
\Lambda=R_{1} \frac{(1-\eta)^{2}}{\eta}+R_{2}(1-\eta)\left|v_{c, f}\right|
$$

and $v_{c, f}$ is the relative velocity between the chip and liquor phases. $R_{1}$ and $R_{2}$ are parameters estimated from experimental data. The pressure distribution is calculated as,

$$
\frac{\partial p_{f}}{\partial z}=-v_{f} \frac{\partial}{\partial z}\left[\rho_{f} v_{f}\right]-\eta v_{f} \frac{\partial \rho_{f}}{\partial t}+\rho_{f} g+\frac{F_{\Lambda}}{\eta}
$$

The digester is assumed to be completely filled with liquor with a fixed pressure on the top. Equation (25) then provides the liquor pressure in the remainder of the digester column.

\section{Chip Phase Velocity Distribution}

In addition to the interphase flow resistance, Härkönen (1987) also used experimental data to model the chip pressure discussed previously as a function of degree of cooking measured by Kappa \# and compaction of chips, $\eta$ as follows,

$$
\begin{aligned}
p_{c} & =10^{4}\left(\frac{(1-\eta)-0.356}{\alpha-0.139 \ln (K \#)}\right)^{1.695} \text { if }(1-\eta)<0.356 \\
& =0, \quad \text { otherwise }
\end{aligned}
$$

where the parameter $\alpha$ has a nominal value of 0.831 based on experimental data. The equation is based on the cross-sectional area of the digester. For chip compaction below 35.6\%, the chips float freely and do not exert pressure on adjacent chips. For a fixed compaction, the chip pressure decreases with increased cooking which corresponds to softening of the chips. The chip pressure also decreases with decreasing volume fraction of chips.

Härkönen (1987) models the force of sliding friction between the chip phase and the digester walls as,

$$
F_{\mu}=\frac{4 \mu p_{c}}{D_{c v}}
$$


where $\mu$ is the coefficient of sliding friction and $D_{c v}$ represents the diameter of the digester cross-sectional area. Using Newton's second law and combining the above forces, we get the axial chip velocity distribution as follows,

$$
\rho_{c}(1-\eta) \frac{\partial v_{c}}{\partial t}=-v_{c}(1-\eta) \rho_{c} \frac{\partial v_{c}}{\partial z}+\rho_{c}(1-\eta) g-\frac{\partial p_{c}}{\partial z}-(1-\eta) \frac{\partial p_{f}}{\partial z}-F_{\Lambda}-F_{\mu}
$$

Pulp production rate in the blowline fixes the boundary condition for the chip momentum equation. Coefficient of viscous friction, chip pressure and the coefficient of sliding friction in equations (24), (26) and (27), respectively are determined empirically. Further experimental work is necessary to determine their accuracy in different operating ranges.

\section{Chip Level}

Chip level constitutes one of the most important variables in digester control. It impacts the Kappa \# via residence time of the chips in the digester. To model chip level, a top section of the digester is assumed, which covers a certain volume over the impregnation zone and consists of the chip phase interface. Chip phase inventory description then provides the dynamic description of level,

$$
\dot{h}_{c}=\frac{1}{A\left(1-\eta_{\text {top }}\right)}\left(\dot{V}_{c, \text { feed }}-A\left(1-\eta_{0}\right) v_{c, 0}\right)
$$

where $\eta_{t o p}$ is an estimate of the compaction in the top section. Michelsen (1995) suggests using a linear relationship between the chip level and $\eta_{\text {top }}$,

$$
\left(1-\eta_{\text {top }}\right)= \begin{cases}0.356 & \text { if } h_{c}<h_{\min } \\ 0.356+k_{t s} h_{c} & \text { otherwise }\end{cases}
$$

where $h_{\min }$ represents a reference height of the chip plug. $\eta_{t o p}$, also forms the boundary condition for Equation (21). Note that variables related to the bottom of the top section are indexed with zero, implying the beginning of the main section of the digester. The coefficient $k_{t s}$ is determined empirically. Equations (21) through (30) are primarily based on works by Härkönen (1987) and Michelsen (1995).

\section{Numerical Method}

For solution purposes, the model PDEs are replaced with a finite difference mesh on a digester grid, such as shown in Figure 3. Notation $j$ refers to a fixed grid node on the digester vessel with the $j^{\text {th }}$ control volume lying between 
nodes $j-0.5$ and $j+0.5$. Note that continuity balances are performed at half nodes while momentum balances for chip and liquor phase are performed at node $j$ with $j-0.5$ and $j+0.5$ as the control volume boundaries (see Figure 3 ). This overlapping of mass and momentum control volumes leads to a staggered grid approach and is routinely used in numerical fluid flow problems (Patankar, 1980). The approach ensures that only reasonable (non-wavy) pressure distributions are acceptable as solutions. In the current model, axial transport of properties in the digester occurs by convection alone, resulting in a system of hyperbolic PDEs. It is well known that solutions based on finite differences distort the information that is being convected downstream (Anderson et al, 1984). For example, simulation of a step-like change upstream of a flow system will exhibit dissipation or dispersion due to the truncation errors in the finite difference scheme, regardless of the modeling assumptions (see Figure 4). To study the impact of the truncation errors, the spatial derivatives were differenced using two different schemes, namely upstream differencing,

$$
\frac{d u_{i}}{d z}=\frac{u_{i}-u_{i-1}}{h}+O(z)
$$

and a fourth order difference formulation,

$$
\frac{d u_{i}}{d z}=\frac{-u_{i-3}+6 u_{i-2}-18 u_{i-1}+10 u_{i}+3 u_{i+1}}{12 h}+O\left(z^{4}\right)
$$

where $h$ represents the height of the finite control volume. Simulation results presented in the next section make use of the first order formula. An assessment of using the higher order formula is presented separately.

\section{Simulation Results and Discussion}

The simulations presented in this section are based on operating parameters presented in Table 5 in Wisnewski et al. (1997). The digester was divided into 96 control volumes and equations (1) through (30) were implemented using Matlab/Simulink on a Sun Sparc Ultra 10,333MHz workstation. Table 1 summarizes the locations of the heater and extraction screen. Inclusion of momentum conservation makes the system relatively stiff. However, computational burden is alleviated by use of the sparsity pattern of the Jacobian matrix. A typical run simulating 80 hours of digester operation requires about 17 minutes of real time. 


\section{Steady State Behavior}

A direct benefit of including momentum in the extended Purdue model is ability to simulate production rate changes. Figure 5 shows comparisons between steady-state profiles of various digester variables for a low production rate (LPR) at $1.328 \mathrm{~m}^{3} / \mathrm{min}$ and a high production rate (HPR) of pulp at $1.478 \mathrm{~m}^{3} / \mathrm{min}$ (dashed line). The blowline flowrate step change was implemented using a first order filter with a time constant of 13 minutes. A simultaneous step change was introduced in the chip flowrate at top of the digester. This was necessary due to the integrating behavior of level dynamics. The reduced residence time of chips leads to lower extent of conversion of lignin (Figure 5a) and a corresponding increase in Kappa \# profile (Figure 5f). The Kappa \# for both the cases shows an initial increase in the impregnation zone $(z<0.14)$ due to rapid consumption of araboxylan relative to lignin. Subsequently, the Kappa \# decreases in the cooking zone $(0.14<z<0.75)$, beyond which the reaction is quenched by the cooler and dilute wash liquor. Figs. 5b-5d show components of entrapped and free liquor. The increase in blowline flow for the HPR case reduces available time for diffusion of EA and HS from free liquor to the entrapped phase leading to a drop in concentrations in the entrapped phase. However, this increases the concentration driving force, which in turn causes the free liquor concentrations to drop as well. These figures also illustrate that the use of the correlation in Equation (15) for calculation of diffusion coefficient leads to nearequilibrium conditions between the free and entrapped liquors. Volume fraction of liquor for the HPR case (Figure $5 \mathrm{~g}$ ) increases in the vicinity of the extraction screen, implying loose packing of chips. This is a result of lower degree of cooking or higher Kappa \# and is consistent with the argument that a greater degree of cooking leads to denser packing of chips. The higher value of $\eta$ results in lowering of liquor velocities (Figure 5i), as more area is available for the free liquor flow. The increase in chip velocity (Figure 5h) is a result of higher volumetric throughput. Figs. $5 \mathrm{j}, \mathrm{k}$ show the temperatures of chip and liquor along the length of the digester. Chip porosity profiles are shown in Figure 51. Chip porosity increases in the direction of chip flow as the solid components are progressively consumed. Relatively lower porosity is observed for the HPR simulation due to lower extent of delignification.

\section{Dynamic Response}

Dynamic behavior of the digester is characterized by large dead times in primary measurements, such as the endpoint Kappa \#. Incorporation of digester hydraulics involves the relatively fast response variables of chip and 
liquor velocities. Changes in liquor flowrates are instantaneously propagated in direction of flow due to the static nature of Equation (22). This difference in time-scales introduces stiffness in the model. Fig 6 shows results for filtered step changes in (1) blowline flow, (2) cooking circulation upper heater, (3) white liquor and (4) dilute liquor flows of $0.15 \mathrm{~m}^{3} / \mathrm{min},-10{ }^{\circ} \mathrm{C},-0.15 \mathrm{~m}^{3} / \mathrm{min}$ and $-0.15 \mathrm{~m}^{3} / \mathrm{min}$, respectively. In each of the cases, the relation between the endpoint Kappa \# and chip level is apparent, as they are coupled via residence time. Cases 1) and 3) result in lower cooking due to lower rate of availability of chemicals, while lower cooking in case 2) is due to lesser amount of energy. The decrease in the chip level in case 4 is due to the lower "lift" provided by the reduced dilute liquor flowrate. In each of the four cases, changes in levels are due to rearrangement of the chip compaction profile. Cases 2) and 3) show kinks and oscillatory behavior in the endpoint Kappa \# plot while traversing from the initial steady state to the final steady state. No simple mechanistic explanation emerges from the dynamic equations. Upon an analysis of simulations, it is observed that the oscillations first appear in the Kappa \# and chip velocity when the upper heater temperature is perturbed (Figure 7). The initial increase in Kappa \# is accompanied by a retardation of chip velocity due to increased chip pressure, which propagates downstream giving rise to the oscillatory behavior in the Kappa \#. Increasing numerical tolerances does not cause this behavior to disappear. Figure 8 shows comparisons for steps in white liquor and dilute liquor flows in opposite directions. Increase in white liquor amounts to increased availability of chemicals and therefore more cooking. For opposite directions of the same-sized step, the process gains are seen to be nonlinear.

\section{Incipient Digester Plugging}

Strong interactions between the mass/energy conservation and the hydraulic features enable simulation of a fault scenario in the digester with serious adverse economic consequences, namely, plugging of the digester. The momentum equation interacts with the model kinetics through the chip pressure gradient, which is a function of the Kappa and compaction profiles. The resulting interaction between the Kappa \# and chip velocity was also evident in Figure 7. Simulation studies reveal that at lower Kappa numbers, chip compaction, (1- $\eta$ ), can significantly increase and chip velocities decrease in the vicinity of the extraction screens forming a plug (see Equation (21)). Chip velocities in the digester usually reduce spatially from the top of the digester to the extraction screen (see for example, Figure 5h). The corresponding softening of the chips increases chip pressure gradient, a negative driving force of the chip velocities in Equation (28). Also increasing flow resistance reduces the value of $\eta$. Since $\eta=0$, 
results in a singularity, only incipient plug formation can be observed (very small values of $\eta$ ), at which point the equations become extremely stiff and the simulation slows down considerably. As an example, steady-state compaction and Kappa \# profiles for a filtered step increase in white liquor flowrate are shown in Figure 9. Excessive cooking nearly causes the digester to plug up near the extraction screen. This is the result of dense packing of cooked chips $(<2 \%$ free liquor) near the extraction screen. The counter flow of dilute liquor aids in dispersing the fibers as the pulp enters the wash zone.

As discussed in the next section, the plugging behavior is found to be sensitive to the parameters in the chip pressure Equation (26). It is possible to eliminate the plugging behavior for the same operating conditions and chips cooked to nearly the same endpoint Kappa \# by changing parameters of the chip pressure equation. While the potential for plugging the digester, when cooked to lower Kappa numbers is encountered in the industry, it may be necessary to perform experiments to further investigate the impact of the chip pressure term on this important fault condition.

\section{Model Parameter Sensitivity Analysis}

Wisnewski et al. (1997) studied model sensitivity to various parameters in the heat and mass transfer equations. Similar trends were observed with the current thermal-hydraulic Purdue model and corresponding results are therefore omitted. Only model sensitivity to some key parameters in the momentum equations is discussed. Figure 10a shows steady state compaction profiles for two different values of the parameter $\alpha$ in Equation (26). Reducing the value of $\alpha$ to 0.816 from the nominal value of 0.831 increases the value the fraction of liquor, $\eta$, from 0.08 to 0.18 , implying lowering of chip compaction at the extraction screen. As discussed in the last section, a very small value of $\eta$ can be interpreted as incipient plugging of the digester. The corresponding Kappa \# profile is shown in Figure 10b, where the endpoint Kappa \# increases by about 3. Thus, the value of the parameter $\alpha$ plays an important role in determining axial distribution of chip compaction in the digester while affecting the Kappa \# profile only marginally.

Figure 11 shows the velocity and compaction profiles when parameter $R_{2}$ in Equation (24) is varied by $30 \%$ in either direction. An increase in $R_{2}$, leads to increased flow resistance, thereby decreasing chip velocities. Consequently, chip compaction rearranges itself by decreasing area available for chip flow (by increasing $\eta$ ) to satisfy overall chip 
continuity. Reducing $R_{2}$, on the other hand, increases has an opposite effect. Negligible changes are observed in the Kappa \# profile. A similar observation is made with flow resistance related parameters $R_{1}$ and $\mu$ in equations (24) and (27), respectively.

\section{Assessment of Higher Order Numerical Method}

To study the impact of using a higher order numerical method on variables of interest, such as endpoint Kappa \#, the following experiment was performed. The simulation based on the first order difference method (Equation (31)) was allowed to reach steady state after which the fourth order scheme (Equation (32)) was used without changing any operating conditions or model parameters. Figure 12 depicts the results. It is noted that the higher order formulation predicts increased conversion of lignin leading to a drop in Kappa \# by about 3. Use of the first order method approximates the digester as a series of CSTRs. However, chip flow in the digester more closely resembles plug flow since backmixing is absent. It is known that the CSTR usually accomplishes lower conversion for a given volume than the plug flow reactor (Fogler, 1999). Thus, the increased conversion with the higher order method is consistent with the fact that the fourth order finite difference formula provides a better approximation of the plug flow behavior in the digester. However, use of the fourth order method decreases system sparsity resulting in increased computation time. Thus, the first order method is retained in all future simulations.

\section{Grade Transition Modeling}

Pulping mills often switch the feedstock grade from hardwood to softwood and vice versa to satisfy demands from the downstream paper machine section. Frequent changes from one feedstock species to other commonly performed in the pulp industry. Of particular interest is reduction of off-spec "twilight" pulp produced during the transition achieved by an effective grade transition policy. Industrial experience indicates that minimizing blending of the feedstock grades at the feed results in "good" transitions. Christensen et al. (1982) incorporated capability to simulate hardwood/ softwood swings by empirically calculating the transition front between the two grades. Their model switched the old grade kinetic parameters to the new grade parameters as the old grade depleted from a finite difference volume and filled with the new grade. The model performed well when compared to swing data. Doyle et al. (2001) modified the extended Purdue model to simultaneously accommodate both, hardwood and softwood species, in the same section of the digester. This was achieved by redefining certain mass concentrations and 
introducing a mixing parameter distributed along the length of the digester. The mixing parameter served as a first order filter which allowed a smooth changes in the mass fraction between the two species.

The current work follows the idea of a discrete transition front as in the Purdue model by Christensen et al. However, unlike their use of an empirical calculation of the grade transition front, momentum conservation allows calculation of the transition front using rigorous fundamental principles. The model assumes that a discrete transition front enters the top of the digester and proceeds downwards. Location of the transition front is calculated by integration of the front velocity obtained from the chip velocity distribution in the digester,

$$
\frac{d z_{0}}{d t}=v_{\text {chips }}\left(z=z_{0}\right)
$$

All kinetic parameters upstream of $z_{0}$ are switched to new grade properties while old grade parameters are retained downstream. Properties within the control volume with the transition front are volume averaged based on the location of the front. Thus, the filtering capability similar to Doyle et al. (2001) exists in the vicinity of the front.

Figure 13 shows Kappa \# profile snapshots at different times during a feed transition from softwood to hardwood. The grade swing was obtained with an open loop operating policy that involved decreasing the upper and lower heater temperatures from $429 \mathrm{~K}$ to $416 \mathrm{~K}$ and $443 \mathrm{~K}$ to $424 \mathrm{~K}$, respectively, as the front location, $z_{0}$, reached the respective heater locations. Also the white liquor flowrate to the top of the digester was decreased by $40 \%$ after the transition front crossed the extraction screens. Current industrial practices also make similar appropriate open-loop manipulations based on front location estimated using the chip meter speed. It is observed that in a softwood to hardwood transition, the reduction in the energy and cooking chemicals, in anticipation of the reduced lignin content of the new grade, results in lower cooking (or higher Kappa \#) during the transition time. On the other hand, transition of the feedstock grade from hardwood to softwood presented in Fig 14, involves increasing temperatures and white liquor flowrates during transition. These actions tend to overcook the remainder of the hardwood grade during transition. Simulation studies show that if the heater temperatures and white liquor flowrates are changed aggressively, the consequent overcooking may result in incipient plugging of the digester. This is consistent with the industrial experience that transition from hardwood to softwood is operationally more difficult than vice-versa. Unlike the softwood to hardwood transition, here the temperatures were increased only after 30 minutes elapsed after the transition front completely crossed the respective heater locations. White liquor flow was increased after 
the transition front crossed the extraction screen. Simulations with heater temperature changes when the front crossed the respective heater location led to the incipient plugging phenomena described earlier. Endpoint Kappa \# transients during grade transitions are shown in Figure 15. The undershoot in the hardwood to softwood transition represents the overcooking of the older hardwood grade. This overcooking takes place despite of the 30 minute delay in increasing the heater temperatures as discussed above. If the heater temperatures are increased as soon as the front reaches the respective heater location, considerable overcooking (endpoint Kappa \# drops to 15 before increasing) and the associated plugging problem are observed. Figure 16 shows the dynamic trajectory of the front location as the hardwood species completely fills the digester. Both the transitions are similar, with softwood to hardwood transition occurring more quickly than the hardwood to softwood transition.

\section{Conclusions}

In the paper and pulp industry, there are vast incentives to reduce off-specification pulp during operation. Unlike the refining industry, very limited blending opportunities exist to produce good quality end products after production. A detailed fundamental model can aid in operational improvements using optimization to evaluate open-loop strategies during production rate and grade transitions. It can also be used to perform system identification and control. Wisnewski and Doyle (1998) show improved control performance using reduced extended Purdue model in comparison to purely data-driven models using model predictive control. However, previous versions of the Purdue model ignored momentum transport, an important element of digester operation. In this work, a thermal-hydraulic model of the continuous digester has been presented. The model provides a detailed description of the important phenomena involved in operating the digester using conservation laws. Constitutive rules were adopted from available literature (Christensen et al. 1982; Härkönen, 1987). Pulping chemistry was based on the Purdue model while the hydraulics mainly rely on work by Härkönen (1987). Thus, the work constitutes a hydraulic extension of the Purdue model. The model illustrates that interactions between the kinetics and hydraulics play an important role in plugging of the digester vessel.

Simulation examples demonstrated various attributes of the model. While no rigorous validation exercise was performed, the variable trends follow those of extended Purdue model (Wisnewski et al., 1997), where applicable, and can be explained mechanistically. The benefits of the hydraulic extension are, however, realized in the ability of 
the model to explain the impact of dynamic flowrate and compaction changes on the pulp quality. Moreover, the chip level, an important control variable is one of the model outputs. Parametric sensitivity revealed that occurrence of incipient digester plugging was strongly related to parameters in the chip pressure equation. Further experimental investigations are necessary to confirm this observation. Incorporation of momentum transport also allows a straightforward extension to modeling grade transitions. An explicit tracking of the transition front allows switching of kinetic parameters. A key contribution lies in demonstrating operational difficulties encountered during a feedstock grade change. It is demonstrated that hardwood to softwood requires delayed changes in heater temperatures and white liquor flowrates to avoid overcooking.

\section{Literature Cited}

Anderson, D.A., J.C. Tannehill and R.H.Pletcher, Computational Fluid Mechanics and Heat Transfer, McGraw Hill, New York (1984).

Christensen, T., L.F. Albright and T.J. Williams A, Mathematical Model of the Kraft Pulping Process, Tech. Rep. 129, Purdue University,PLAIC, West Lafayette, Indiana (1982).

Doyle, F.J., L. Puig, and F. Kayihan, "Grade Transition Modeling in Continuous Pulp Digester for Reaction Profile Control", Pulp \& Paper Canada, 102, 56-59 (2001).

Fogler, H.S, Elements of Chemical Reaction Engineering, $3^{\text {rd }}$ ed, Prentice Hall, New Jersey (1999).

Gustafson, R., C. Sleicher, W. McKean and B. Finlayson, "Theoretical Model of the Kraft Pulping Process", Ind. Eng. Chem. Proc. Des. Dev., 22, 87-96 (1983).

Härkönen, E.J., "A Mathematical Model for Two-Phase Flow in a Continuous Digester", TAPPI J., 70, 122-126 (1987).

He, P., M. Salcudean, I.Gartshore, and E.L. Bibeau, "Modeling of Kraft Two-Phase Digester Pulping Processes", TAPPI Conference, Anaheim, California (1998).

Kayihan, F., M.S. Gelormino, E.M. Hanczyc, F.J. Doyle III, and Y. Arkun, "A Kamyr continuous digester model for identification and controller design", Proc. IFAC World Congress, San Francisco, 37-42 (1996).

McKibbins, S.W., "Application of Diffusion Theory to the Washing of Kraft Cooked chips", Tappi J., 43, 801-805 (1960).

Michelsen, F.A., A Dynamic Mechanistic Model and Model-Based Analysis of a Continuous Kamyr Digester, Ph.D. Thesis, University of Trond heim (1995).

Patankar, S., Numerical Heat Transfer and Fluid Flow, McGraw Hill, New York (1980).

Smith, C.C., and T.J. Williams, Mathematical Modeling, Simulation and Control of the Operation of Kamyr Continuous Digester for Kraft Process, Tech. Rep 64, Purdue University, PLAIC, West Lafayette, Indiana (1974).

Wisnewski, P.A., F.J. Doyle III, and F. Kayihan, "Fundamental Continuous Pulp Digester Model for Simulation and Control”, AIChE J., 43, 3175-3192 (1997).

Wisnewski, P.A., and F.J. Doyle III, "Control Structure Selection and Model Predictive Control of the Weyerhaeuser Digester Problem”, J. Proc. Control, 8, 487-495 (1998). 
Page 22 of 34

\section{Acknowledgement}

The authors acknowledge funding from the Department of Energy (Grant DE-FC07-00ID-13882), Westvaco Inc., and Weyerhaeuser Inc. 
Table 1: Number of control volumes in the different zones of digester

\begin{tabular}{|c|c|}
\hline Digester Zone & Number of Control Volumes \\
\hline Impregnation & 17 \\
Upper Heater & 7 \\
Lower Heater & 7 \\
Cooking & 41 \\
Wash & 24 \\
\hline
\end{tabular}




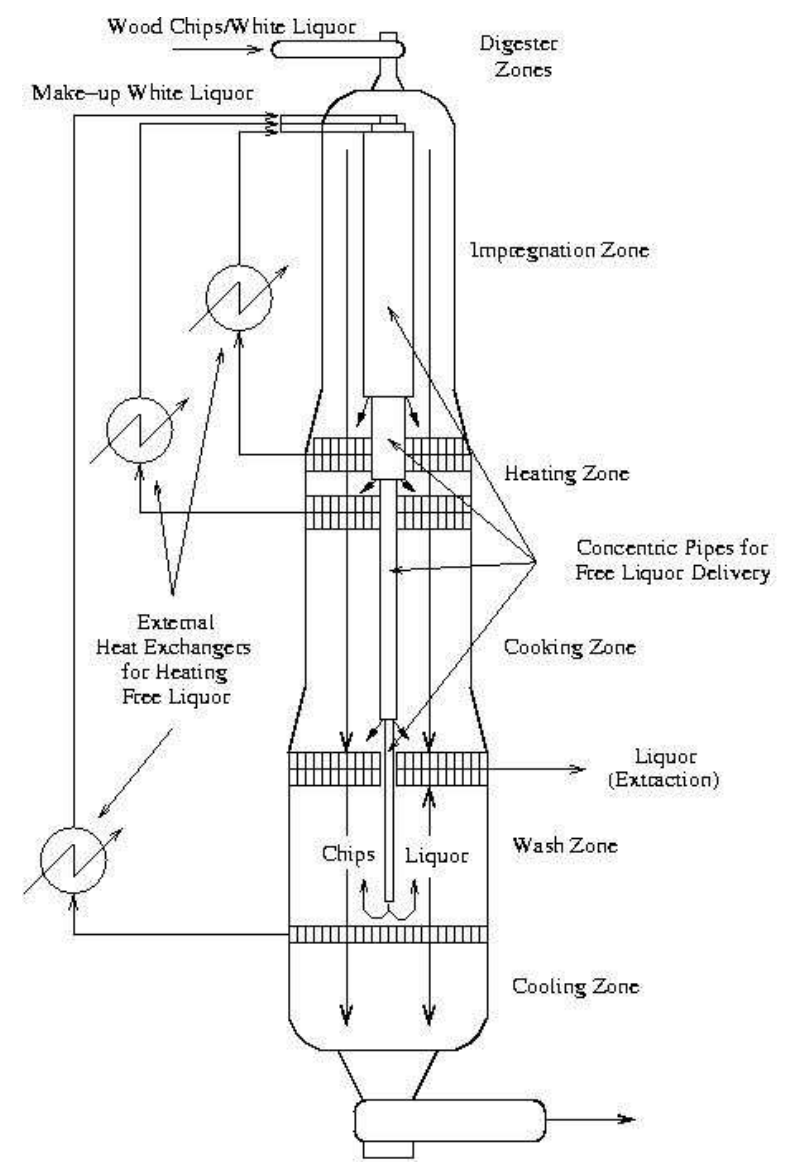

Figure 1. Schematic of a single vessel continuous digester.

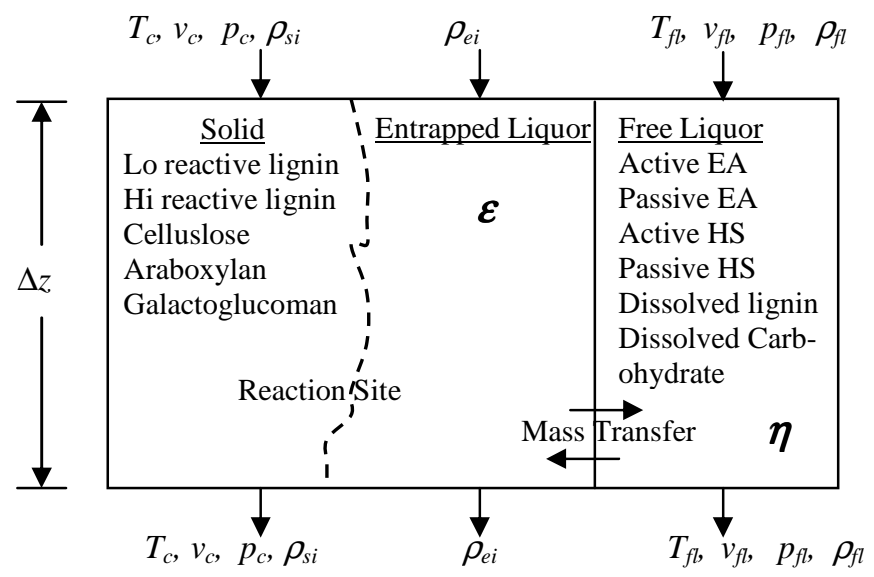

Figure 2. A cross-sectional slice of digester (cook zone) consists of solid, entrapped liquor and free liquor phases. 


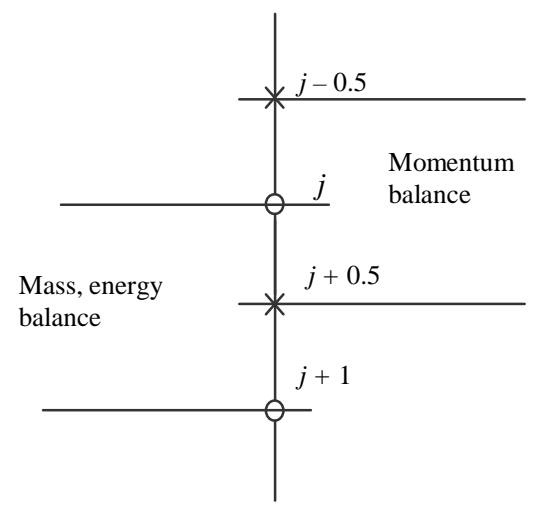

Figure 3. Arrangement of overlapping control volumes in digester vessel.

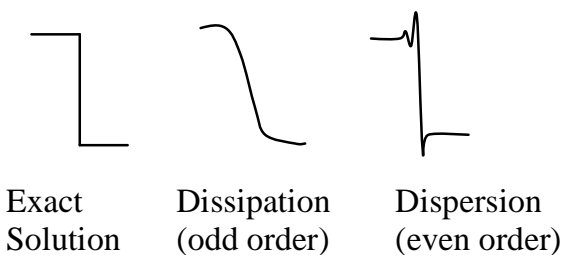

Figure 4. Use of finite differences induce numerical diffusion. 

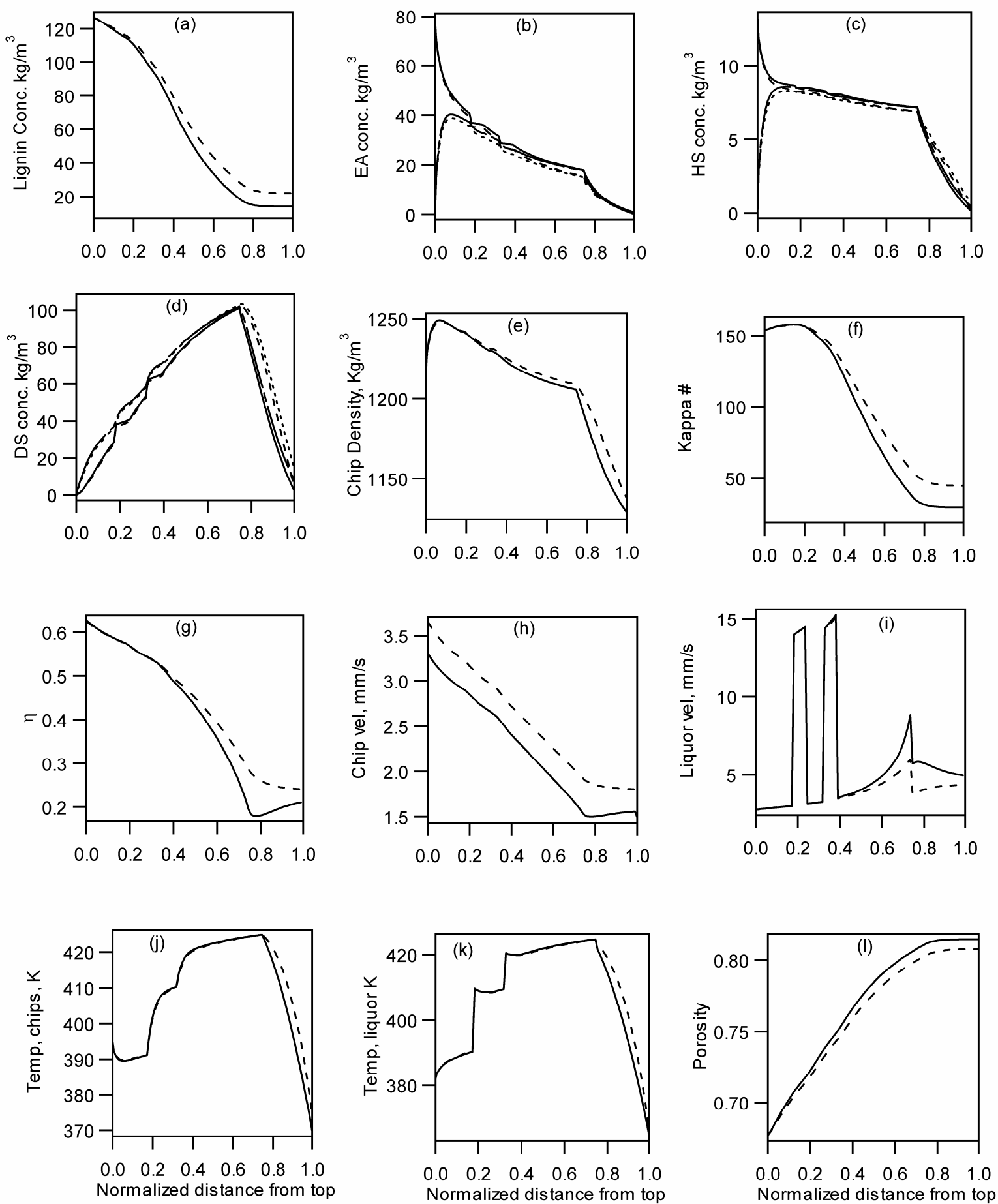

Fig 5. Steady state behavior for low (solid) and high (dashed) blowline flowrates. Impregnation zone: $z<0.14$; : cook zone: $0.14 \leq z,<0.75$; wash zone: $z \geq 0.75$. 
Page 27 of 34
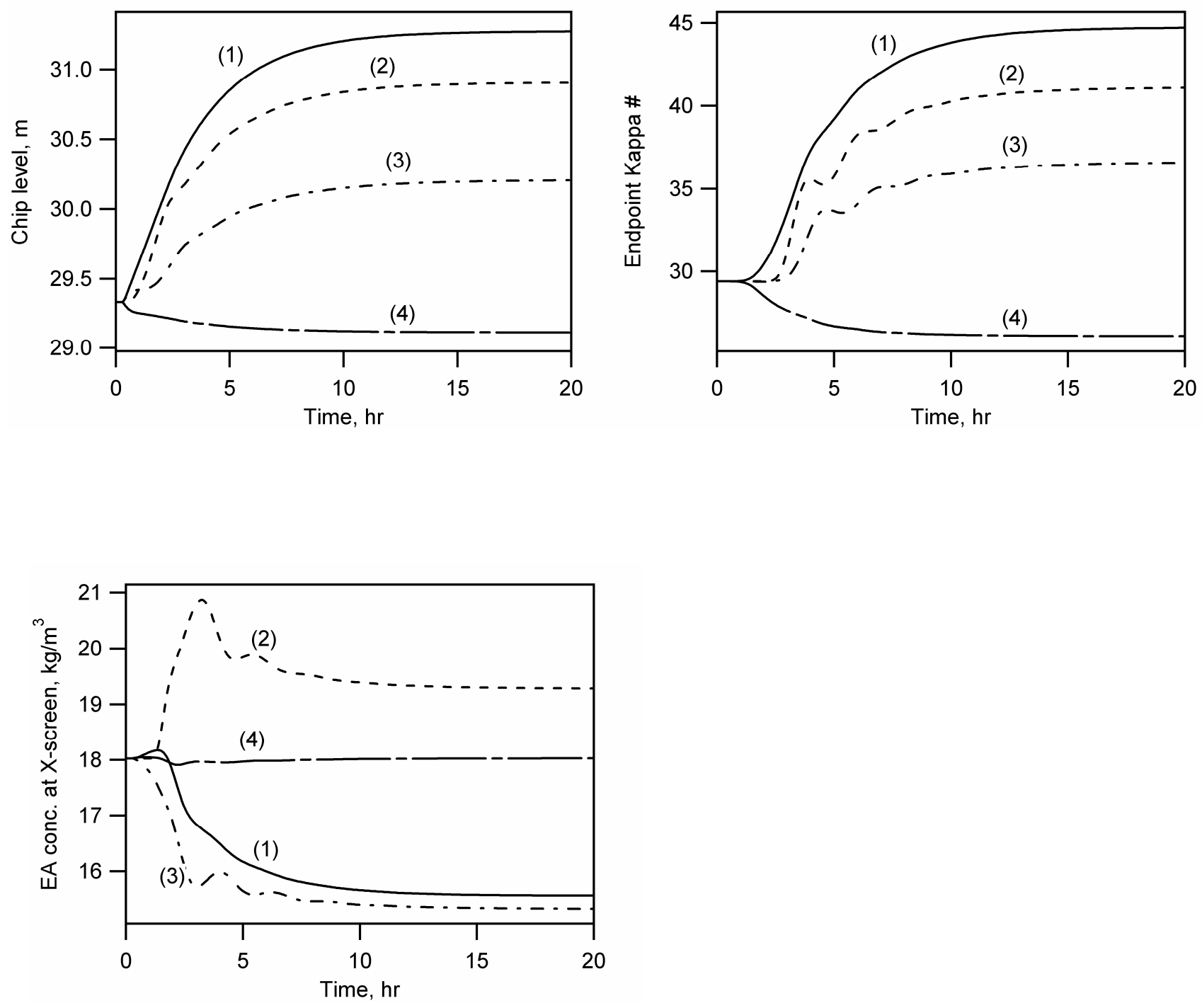

Figure 6. Dynamic behavior for (1) increase in blowline flow; (2) decrease in upper heater temp.; (3) decrease in white liquor; and (4) decrease in dilute liquor flow. 
Page 28 of 34
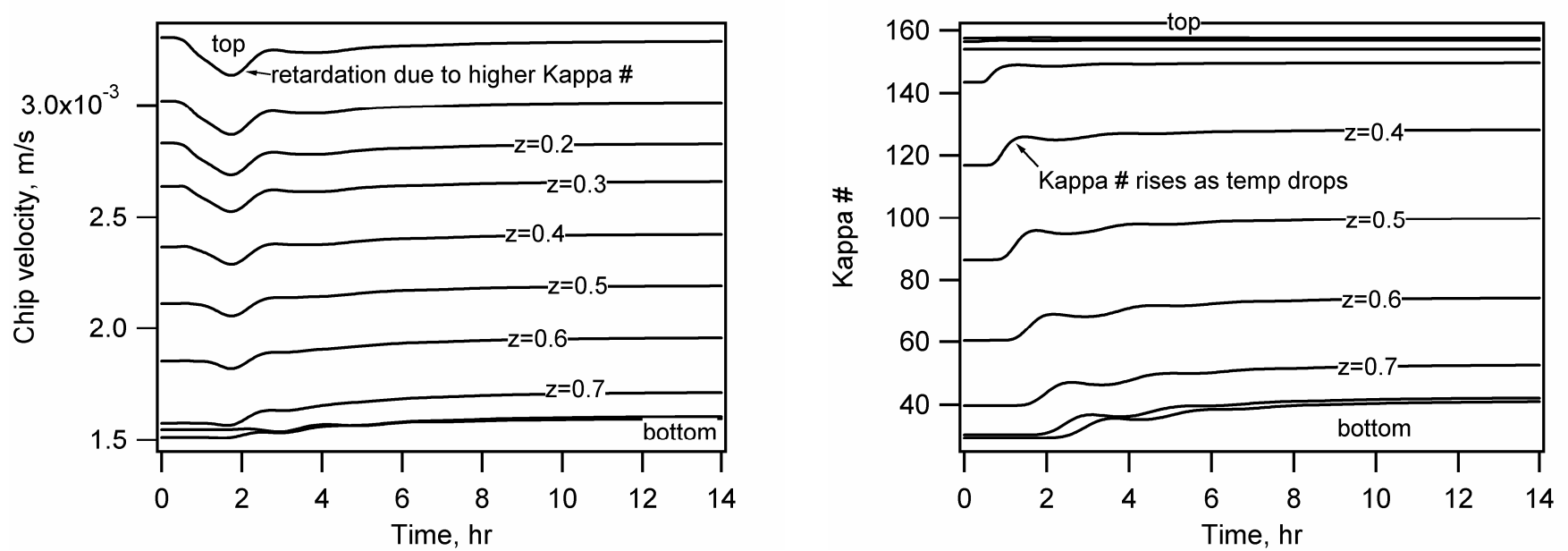

Figure 7. Velocity and Kappa \# transients at various locations on the digester due to change in upper heater circulation temperature ("top" and "bottom" refer to the digester).
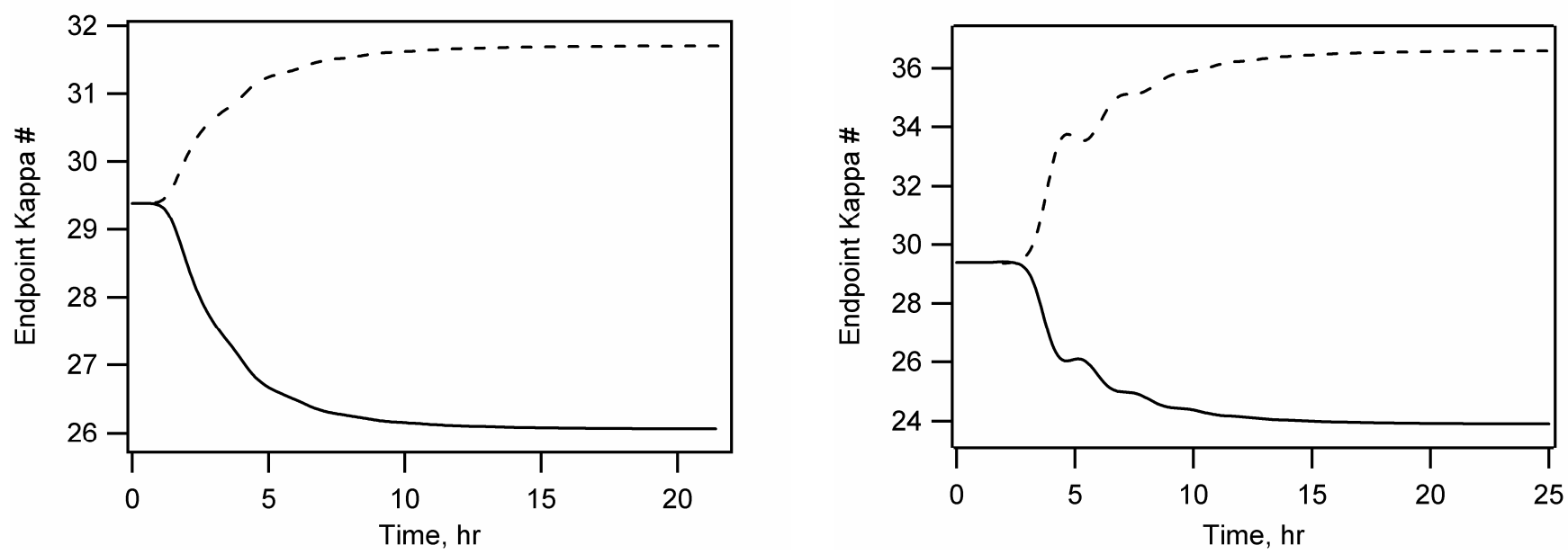

Figure 8. Symmetric step tests for (a) $\pm 0.15 \mathrm{~m}^{3} / \mathrm{min}$ change in dilute liquor flow; and (b) $\pm 0.15 \mathrm{~m}^{3} / \mathrm{min}$ change in white liquor flow. 

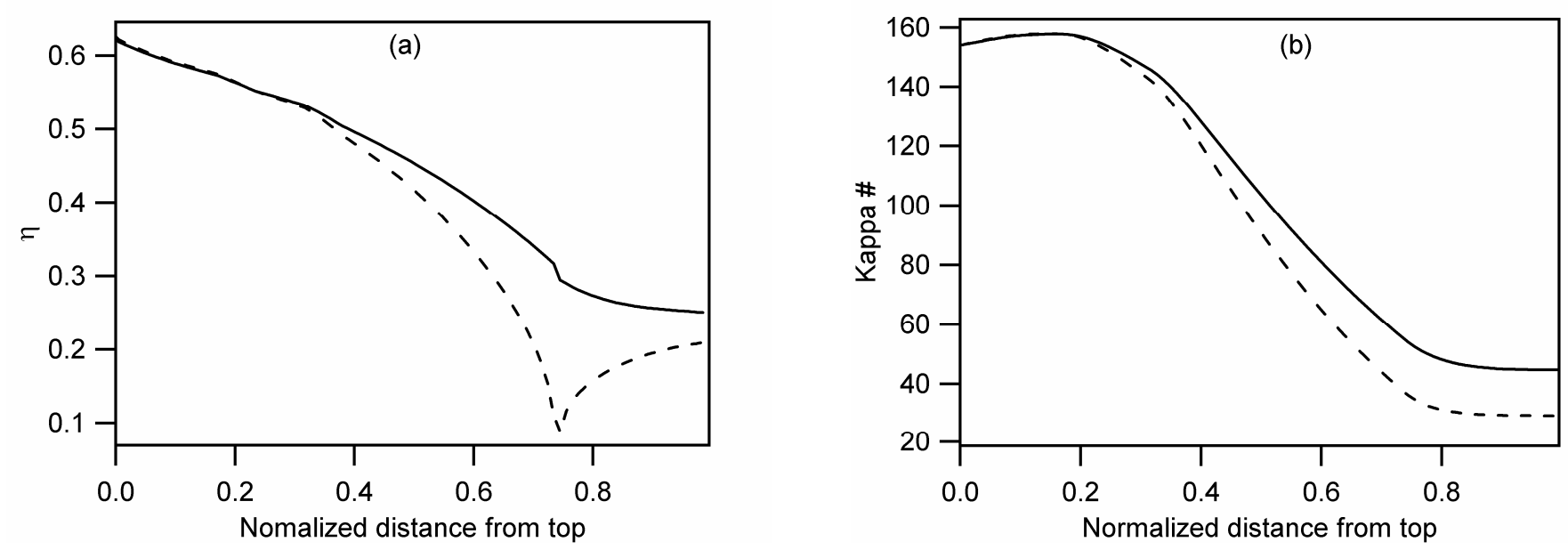

Figure 9. Steady state compaction and Kappa \# profiles for low (solid) and high (dashed) values of white liquor flowrates. The sharp kink in the compaction profile (dashed) corresponding to higher white liquor flow represents incipient plugging in the digester. 
(a)

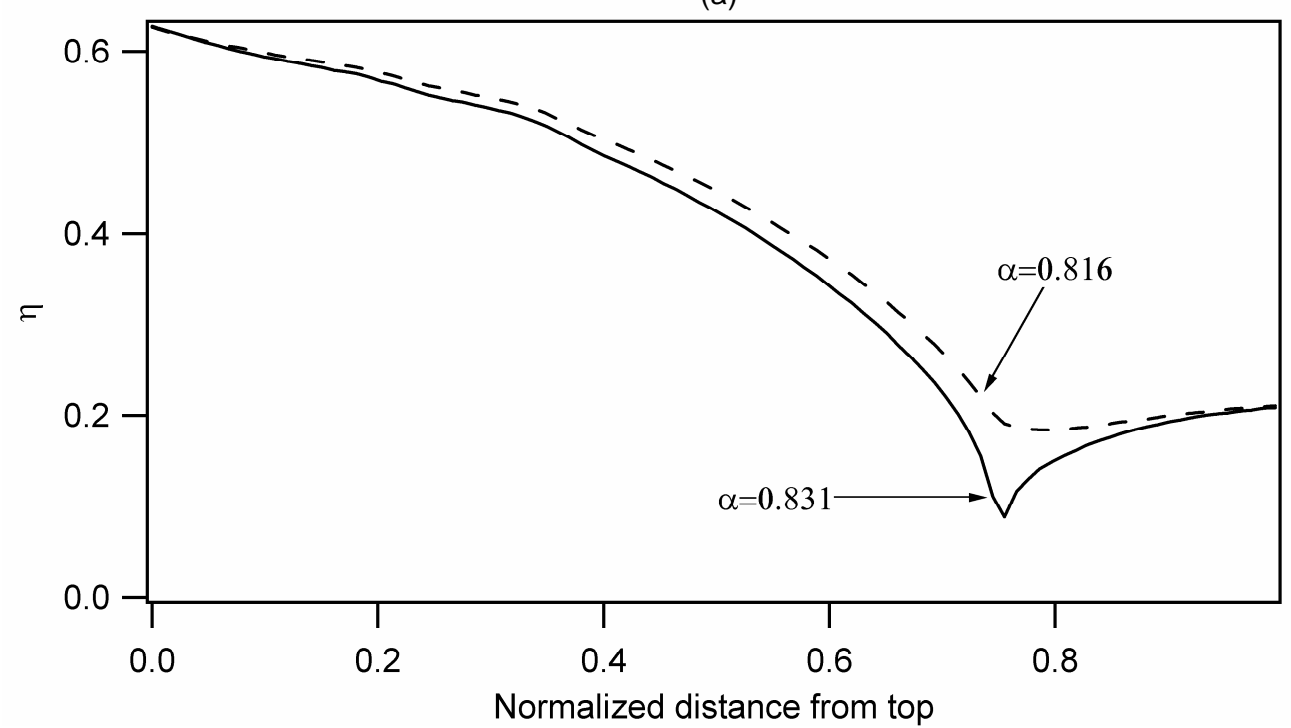

(b)

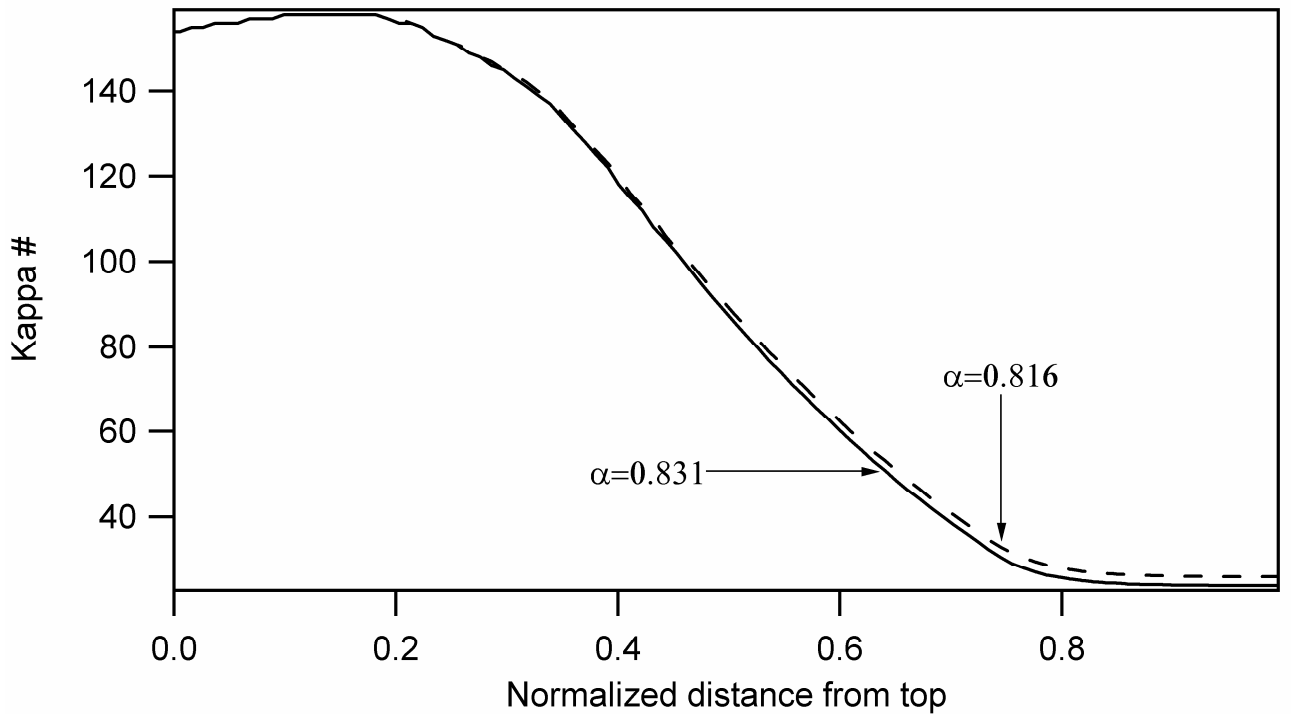

Fig 10. Sensitivity of (a) chip compaction and (b) Kappa \# to changes in parameter $\alpha$ in Equation (26). While no substantial change is observed in the Kappa \# profile, the chip compaction shows chip compaction is lowered when $\alpha$ is changed from the nominal value of 0.831 to 0.816 , implying easier drainage of chips. 
(a)

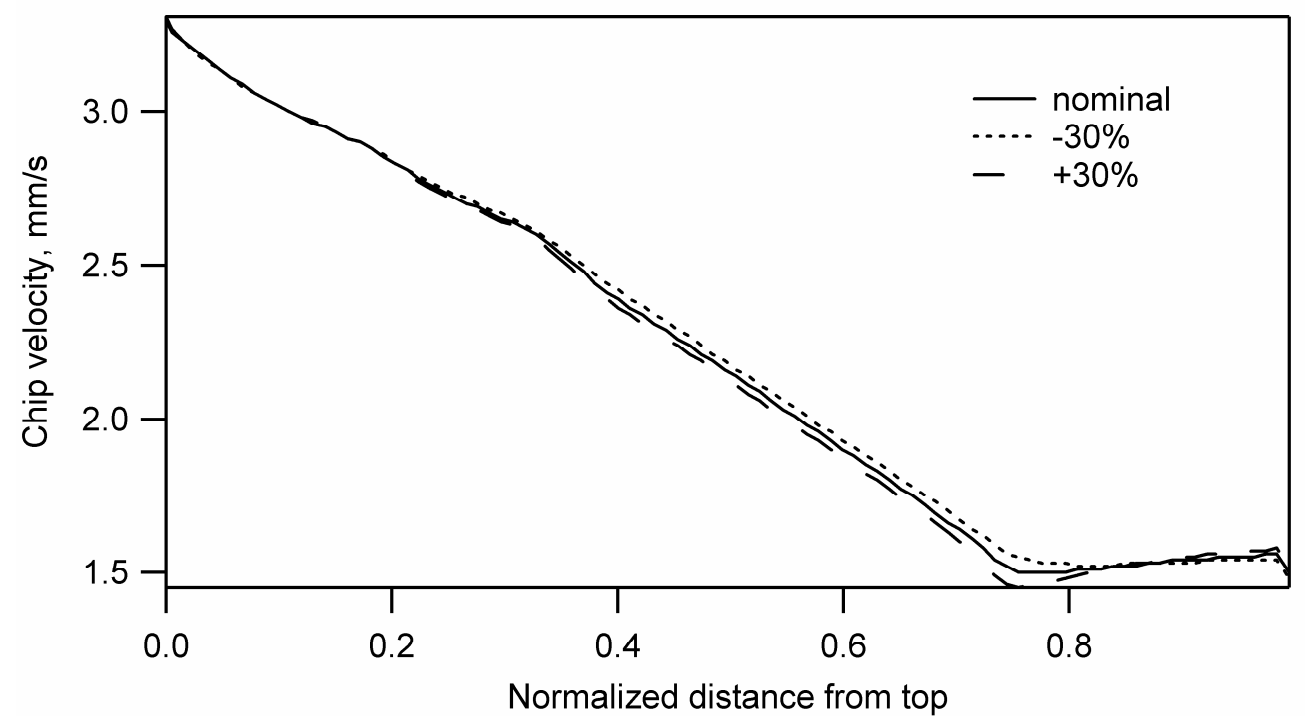

(b)

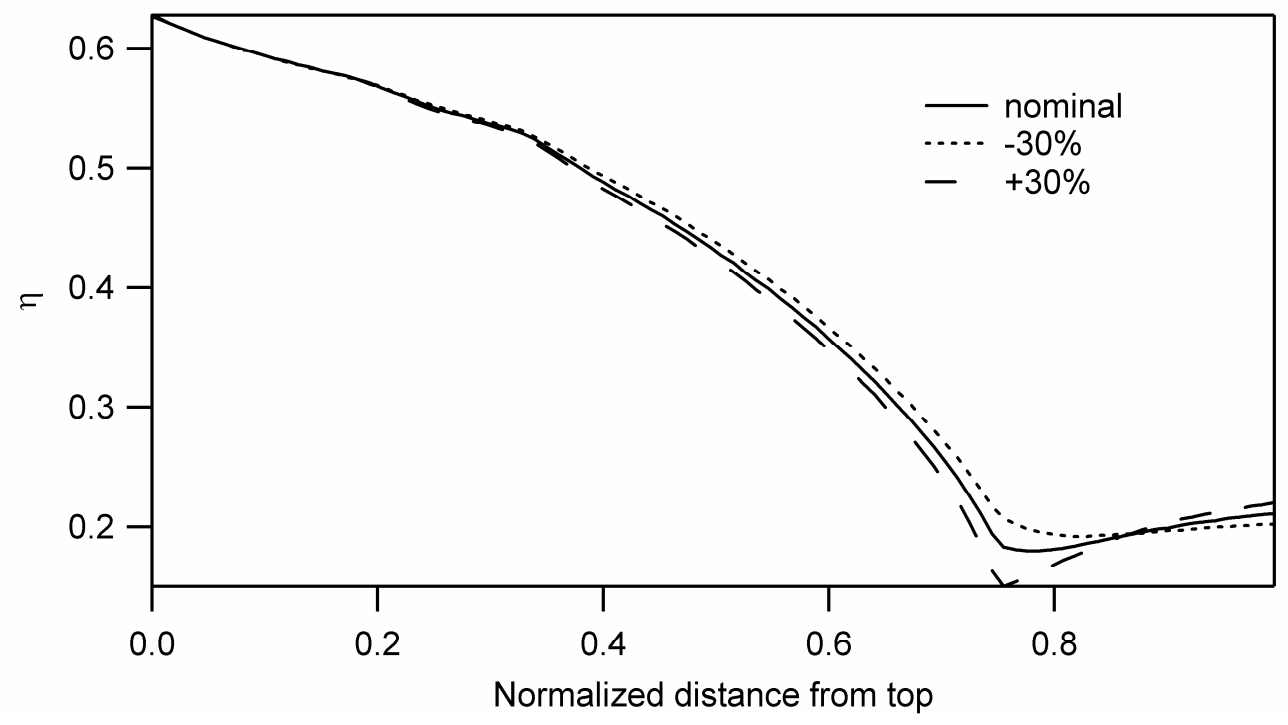

Figure 11. Model sensitivity to change in parameter $R_{2}$ in Equation (24). (a) Increasing $R_{2}$ increases flow resistance and hence causes a decrease in velocity. (b) To maintain the same volumetric flowrate, a corresponding decrease in $\eta$ is observed. 


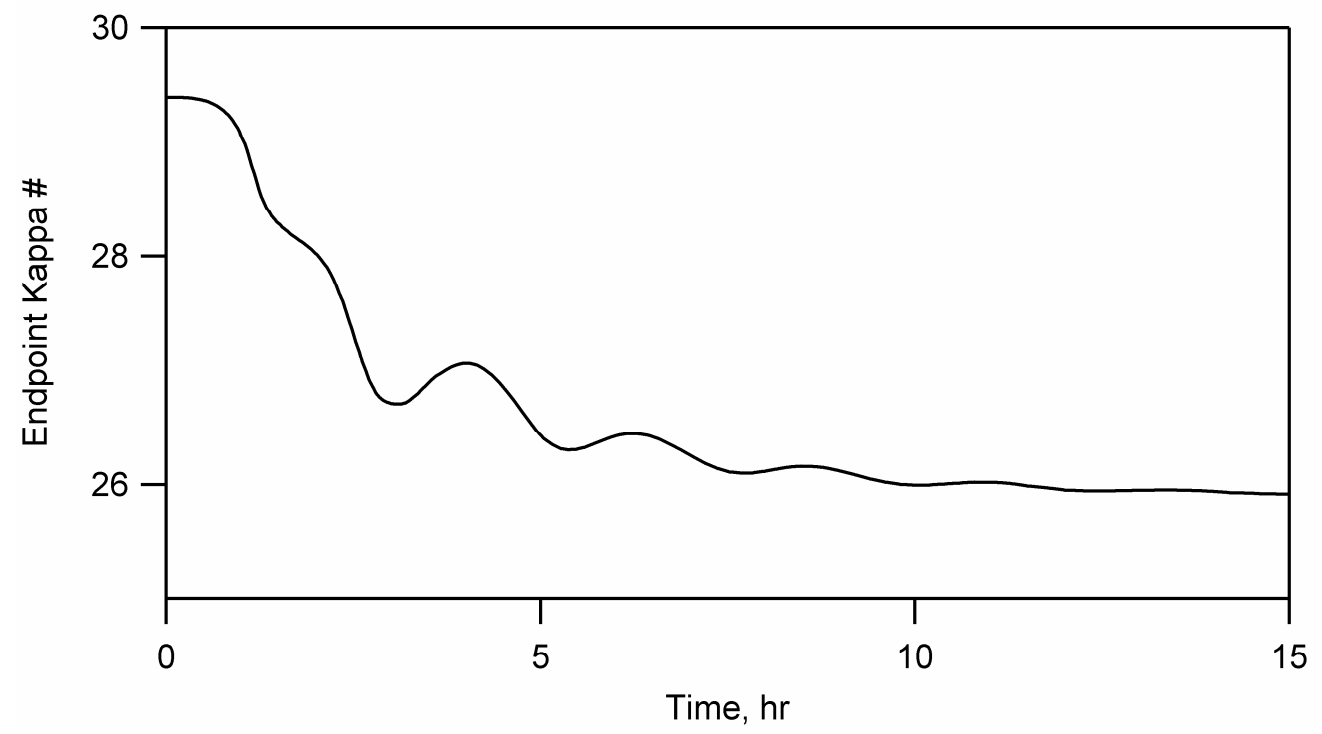

Figure 12. Higher order numerical method predicts higher removal of lignin from chips. The initial conditions correspond to steady-state conditions with a first order finite difference formulation. At $t=0$, the convective flow of chips is described by a $4^{\text {th }}$ order formulation with all operating parameters unchanged.

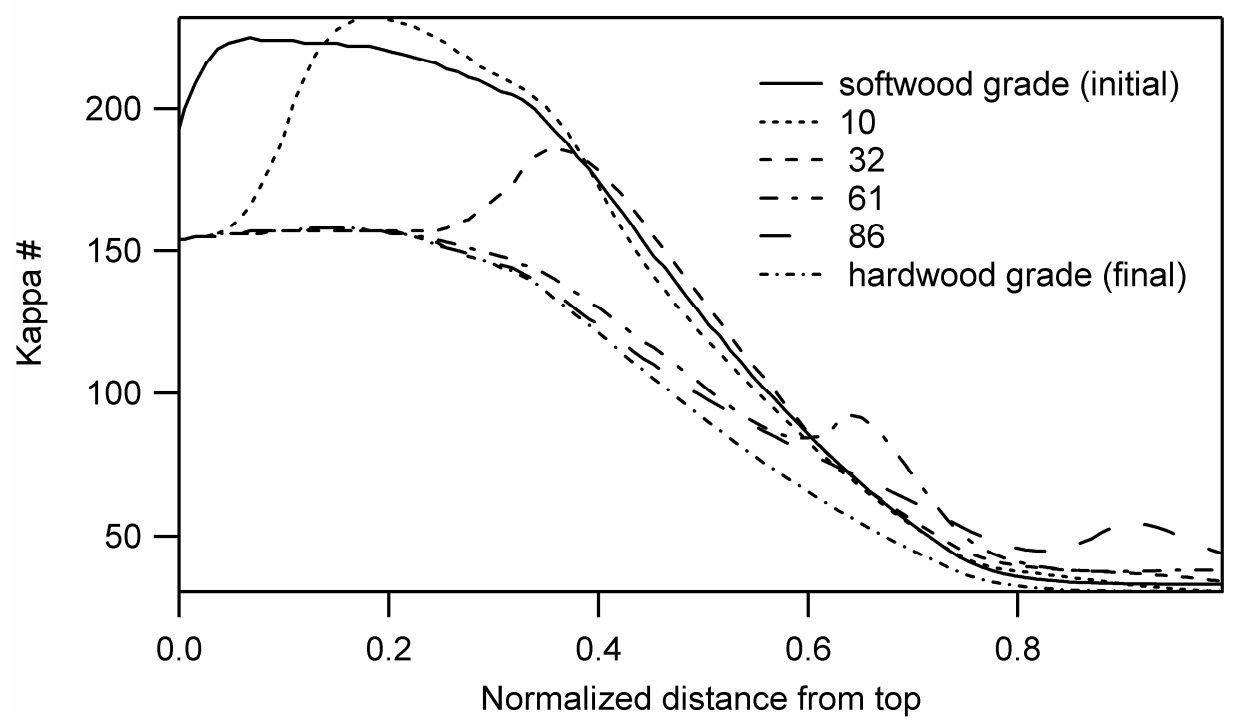

Fig 13. Kappa profile snapshots when the softwood to hardwood transition front is at different locations. The numbers in the legend refer to the control volume in which the front lies when the snapshot is taken. Appropriate changes were made to the heater temperatures to achieve acceptable endpoint Kappa numbers. 


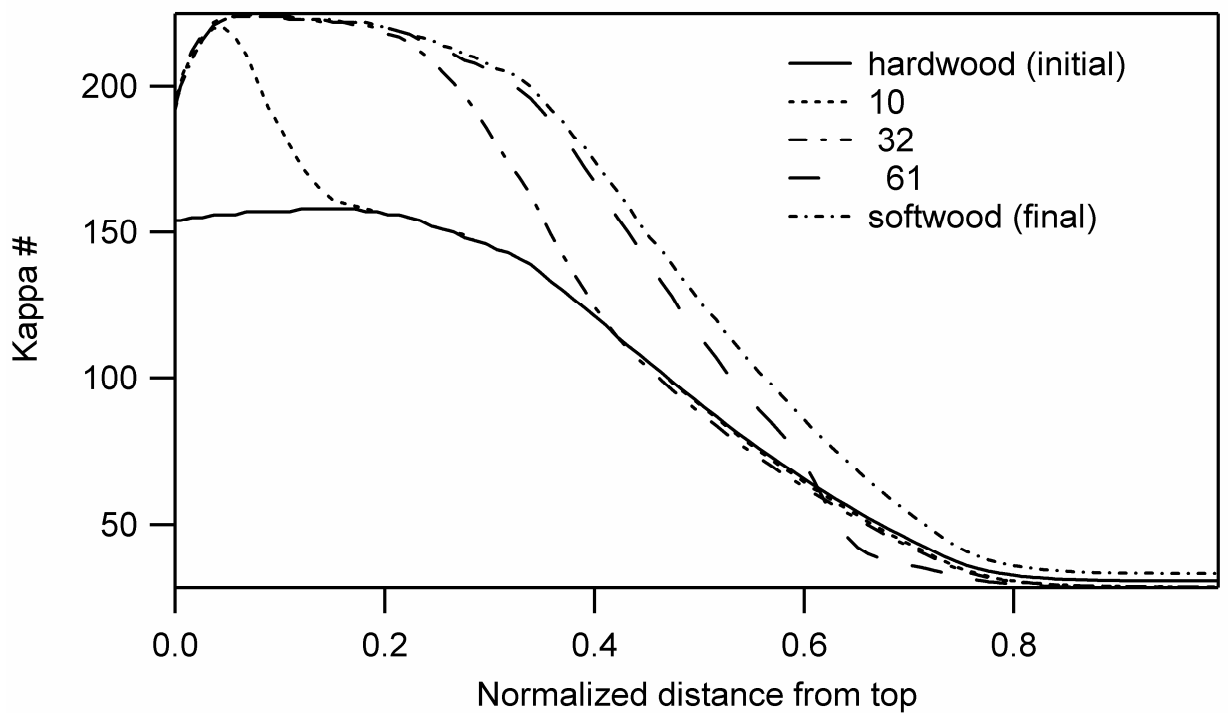

Figure 14. Kappa profile snapshots for hardwood to softwood transition. The numbers in the legend refer to the control volume in which the front lies when the snapshot is taken.

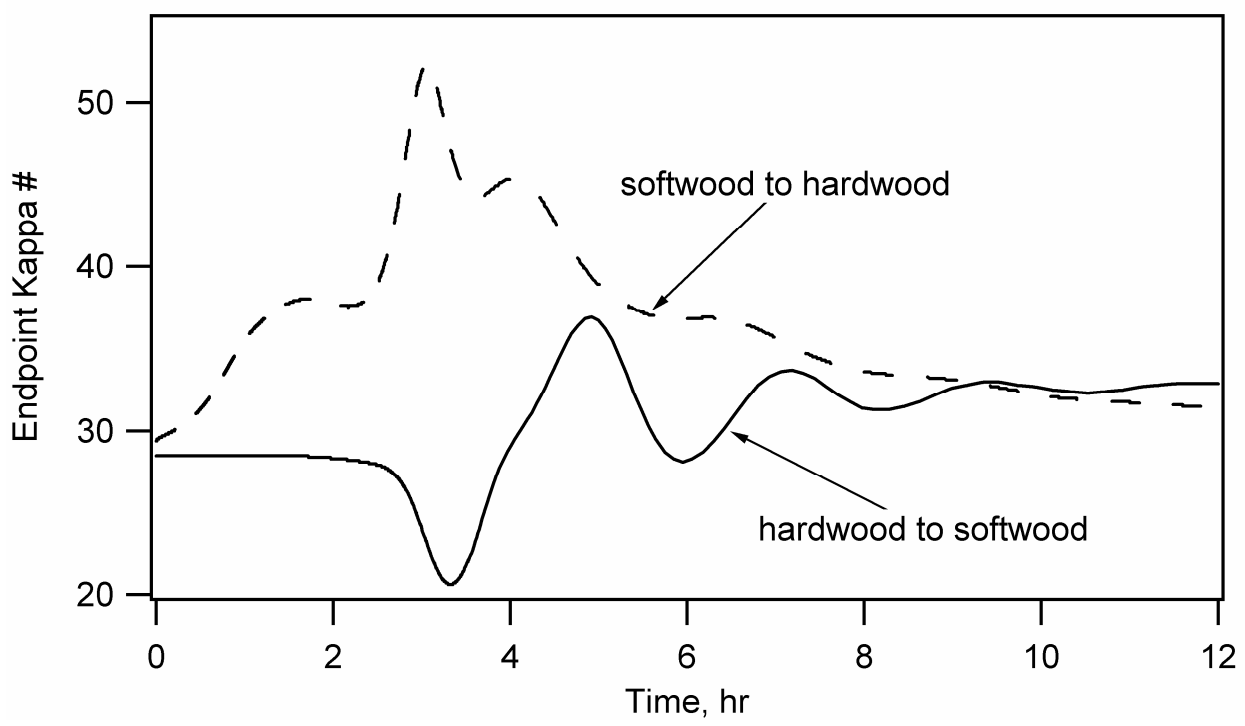

Figure 15. Digester endpoint kappa number transients for grade change transitions. Increase in heater temperatures during the hardwood to softwood transition overcooks the exiting hardwood grade leading to the undershoot in the endpoint Kappa \#. 


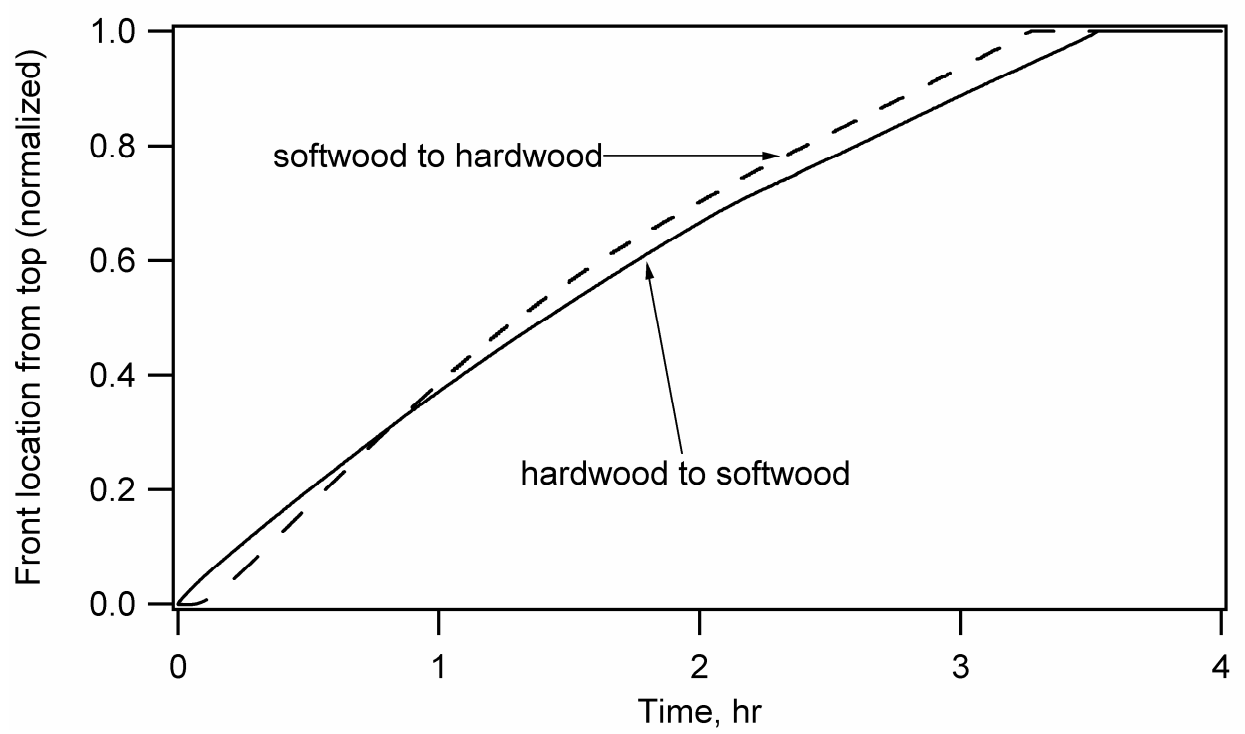

Figure 16. Dynamic trajectory of transition front for the two cases of grade transition. Grade transition is modeled by assuming a discrete transition front. Kinetic parameters upstream of the front are switched to values for the new grade, while old grade parameters are retained downstream. 